\title{
Interferon- $\gamma$ release assays for the diagnosis of latent Mycobacterium tuberculosis infection
}

\section{To the Editors:}

In their meta-analysis, DiEL et al. [1] report a superior performance of interferon- $\gamma$ release assays (IGRAs) for the diagnosis of latent tuberculosis infection (LTBI) compared with the tuberculin skin test (TST), the only other test available for diagnosing LTBI.

In the absence of a gold standard for LTBI, the proper assessment of the TST, IGRAs or any other future tests intended for the diagnosis of LTBI should focus on determining their capability for predicting the development of active tuberculosis (TB), i.e. the positive and negative predictive values for progression to active TB. Alternative evidence, such as the diagnostic accuracy of the test in active TB cases, is not appropriate for assessing the test's capability to predict the development of TB.

Thus far, few studies have assessed the predictive capability of IGRAs and the TST; among them, only the study by DIEL et al. [2] in Germany found IGRAs to be superior to the TST in predicting the development of TB (14.6\% of IGRA-positive subjects versus $2.3 \%$ of TST-positive individuals developed TB during the 24-month follow-up period) [2]. All other studies comparing IGRAs and the TST, including two studies not included in the authors' meta-analyses [3, 4], have reported a very similar rate of progression to active TB, ranging from 1.7 to $3.3 \%$ for IGRApositive and 1.7 to $3.1 \%$ for TST-positive individuals, with follow-up periods from 19 to 24 months. The reasons for the discrepancy between these studies and the original study by DiEL et al. [2] are not clear but could be due to different at-risk populations and, possibly, the type of tuberculin applied, as $0.1 \mathrm{~mL}$ Tuberculin-10-GT (Chiron Behring, Marburg, Germany) was used in an unknown proportion of subjects. The authors do not provide information on the equivalence of this tuberculin to the recommended purified protein derivative-S [5].

The main problem with most of these studies is the small number of study subjects and/or active TB cases developing among contacts: 6-15 TB cases were reported in all studies except one, which reported 26 cases [3].

Most studies that compared IGRAs and TST have reported low overall positive predictive values. Further studies are unlikely to show that IGRAs and the TST have better predictive capabilities because active TB develops in a minority of subjects who are considered to be infected, unless a different test or methodological approach is used.

In a large, longitudinal study in Canada that assessed the predictive value of the TST among untreated contacts of active TB cases, we found that $6.9 \%$ of household contacts and $48 \%$ of contacts $0-10$ yrs of age with a positive TST developed TB [6]. These are much higher rates of progression to active TB than those reported in most studies for contacts with a positive IGRA.

Another potential limitation of IGRAs not considered by DIEL et al. [1] is the high reversion rate (conversion from positive to negative) of $33-49 \%$ reported in studies where individuals were retested with IGRAs within a 1-3-month period [7, 8].

Until better tests are available, the usefulness of the TST and IGRAs, whether alone or in combination, should ideally be assessed by cost-effectiveness studies in different high-risk groups for developing TB: household contacts, contacts $0-10$ yrs of age and immunosuppressed contacts of active TB cases [9]. Future studies are therefore needed to determine whether IGRAs, the TST or both are currently the best option in predicting the risk of developing TB.

\section{O. Moran Mendoza}

Division of Respiratory and Critical Care Medicine, Queen's University, Kingston, ON, Canada.

Correspondence: O. Moran Mendoza, Division of Respiratory and Critical Care Medicine, Queen's University, 102 Stuart Street, Kingston, K7L2V6, Canada. E-mail: morano@queensu.ca

Statement of Interest: None declared.

\section{REFERENCES}

1 Diel R, Goletti D, Ferrara G, et al. Interferon- $\gamma$ release assays for the diagnosis of latent Mycobacterium tuberculosis infection: a systematic review and meta-analysis. Eur Respir J 2011; 37: 88-99.

2 Diel R, Loddenkemper R, Meywald-Walter K, et al. Predictive value of a whole blood IFN- $\gamma$ assay for the development of active tuberculosis disease after recent infection with Mycobacterium tuberculosis. Am J Respir Crit Care Med 2008; 177: 1164-1170.

3 Hill PC, Jackson-Sillah DJ, Fox A, et al. Incidence of tuberculosis and the predictive value of ELISPOT and Mantoux tests in Gambian case contacts. PLoS One 2008; 3: e1379.

4 Bakir M, Millington KA, Soysal A, et al. Prognostic value of a T-cellbased, interferon- $\gamma$ biomarker in children with tuberculosis contact. Ann Intern Med 2008; 149: 777-787.

5 Migliori GB, Raviglione MC, Schaberg T, et al. Tuberculosis management in Europe. Task Force of the European Respiratory Society (ERS), the World Health Organisation (WHO) and the International Union against Tuberculosis and Lung Disease (IUATLD) Europe Region. Eur Respir J 1999; 14: 978-992.

6 Moran-Mendoza O, Marion SA, Elwood K, et al. Tuberculin skin test size and risk of tuberculosis development: a large population-based study in contacts. Int J Tuberc Lung Dis 2007; 11: 1014-1020. 
7 Hill PC, Brookes RH, Fox A, et al. Longitudinal assessment of an ELISPOT test for Mycobacterium tuberculosis infection. PLoS Med 2007; 4: e192.

8 Gandra S, Scott WS, Somaraju V, et al. Questionable effectiveness of the QuantiFERON-TB Gold Test (Cellestis) as a screening tool in healthcare workers. Infect Control Hosp Epidemiol 2010; 31: 1279-1285.

9 Moran-Mendoza O, Marion SA, Elwood K, et al. Risk factors for developing tuberculosis: a 12-year follow-up of contacts of tuberculosis cases. Int J Tuberc Lung Dis 2010; 14: 1112-1119.

DOI: $10.1183 / 09031936.00182710$

\section{From the authors:}

We thank O. Moran Mendoza for his comment on the joint systematic review and meta-analysis on the role of interferon- $\gamma$ release assays (IGRAs) for the diagnosis of latent infection with Mycobacterium tuberculosis by the Tuberculosis Network European Trials Group (TBNET) and the European Centre for Disease Prevention and Control (ECDC) [1]. O. Moran Mendoza highlights the as-yet limited knowledge about the positive predictive values of IGRAs for the development of tuberculosis, a standpoint that is also expressed in the new European Union guidance on the use of IGRAs in support of the diagnosis of tuberculosis [2].

In our meta-analysis and systematic review, only studies reporting on the diagnosis of latent infection with $M$. tuberculosis (LTBI) that used the latest commercially available version of IGRAs were included. Studies that were performed with "in-house" IGRAs, e.g. the studies by HILL et al. [3] and by BAKIR et al. [4], were not considered. Although it was not the focus of our review, differences between the studies with commercially available IGRAs and these two studies should be highlighted. Among the 26 contacts who developed active tuberculosis in the study by HiLl et al. [3] from Gambia, no clear linkage of secondary tuberculosis cases to the putative index cases could be established. In addition, the authors used a slightly different cut-off for IGRA positivity, limiting direct comparison of the results. BAKIR et al. [4] performed a primarily interventional study. Most of the household children and adolescent contacts with a positive tuberculin skin test (TST) or IGRA result received isoniazid preventive chemotherapy for a duration of 6 months. This intervention was $\sim 60 \%$ effective at preventing tuberculosis and, thus, substantially influenced the evaluation of the prognostic value of IGRAs for the development of tuberculosis.

In the European region, two large studies that have addressed the positive predictive value of latest-generation IGRAs for the development of tuberculosis have been published to date. While DIEL et al. [5] found a clear superiority of an IGRA over the TST for the development of tuberculosis in household contacts, KIK et al. [6] found IGRAs and the TST equally ineffective at predicting the development of tuberculosis in immigrants to the Netherlands. As the study populations investigated were different and KIK et al. [6] performed IGRAs only when the TST was positive, the results of both studies are not directly comparable.

In countries of low-to-medium tuberculosis prevalence, shortterm progression rates to tuberculosis in individuals belonging to tuberculosis risk groups have been reported to range from $3.3 \%$ to $12.9 \%$ [5-9]. Although the potential cost-effectiveness of an IGRA for the investigation of LTBI has recently been demonstrated in immigrant screening in the UK [10], information on the predictive values of IGRAs for the development of tuberculosis still relies on a small number of at-risk individuals who actually developed tuberculosis. Clearly, more information is needed before general recommendations that IGRAs should replace the TST can be made.

Future prospective studies that will address the role of immunodiagnosis in tuberculosis risk assessment should focus on stratification of subjects into different risk groups and be performed in multiple centres.

There is a striking difference in the positive and negative predictive values of the TST and IGRAs for the diagnosis of tuberculosis. While the negative predictive values for all tests in clinical use are $>97 \%$, the positive predictive values are low and the large majority of individuals at risk for the development of tuberculosis with a positive TST and/or IGRA result will not develop tuberculosis. Clinical decision makers in many countries are, therefore, hesitant to advocate consequently for preventive chemotherapy despite positive test results. Biomarkers for the prediction of tuberculosis need to be substantially improved beyond the limited performance of the TST and IGRAs, and treatment duration should be shortened substantially. Advocacy of preventive chemotherapy by caregivers and acceptance by individuals at risk for the development of tuberculosis need to be improved. As a general rule, testing should only be offered to those individuals belonging to at-risk groups to identify those with the highest risk for future tuberculosis development.

\section{R. Diel*, D. Goletti ${ }^{*}$, C. Lange ${ }^{\boldsymbol{\tau}}$ and D. Manissero ${ }^{+}$}

*Dept of Pulmonary Medicine, Hanover Medical School, Hanover, and "Division of Clinical Infectious Diseases, Research Centre Borstel, Borstel, Germany. "Division of Translational Science, National Institute for Infectious Diseases L. Spallanzani, Rome, Italy. ${ }^{+}$European Centre for Disease Prevention and Control, Stockholm, Sweden.

Correspondence: C. Lange, Tuberculosis Centre Borstel, Division of Clinical Infectious Diseases, Medical Clinic, Parkallee 35, 23845 Borstel, Germany. E-mail:clange@ fz-borstel.de

Statement of Interest: Statements of interest for R. Diel and D. Goletti can be found at www.erj.ersjournals.com/site/misc/ statements.xhtml

\section{REFERENCES}

1 Diel R, Goletti D, Ferrara G, et al. Interferon- $\gamma$ release assays for the diagnosis of latent Mycobacterium tuberculosis infection: a systematic review and systematic review and meta-analysis. Eur Respir J 2011; 37: 88-99.

2 European Centre for Disease Prevention and Control. Use of Interferon- $\gamma$ Release Assays in Support of TB Diagnosis. Stockholm, European Centre for Disease Prevention and Control, 2011. 